\title{
Direct-to-implant breast reconstruction following nipple-sparing mastectomy: predictive factors of adverse surgical outcomes in Asian patients
}

\author{
Chun-Lin $\mathrm{Su}^{1}$, Jia-Ruei Yang ${ }^{1}$, Wen-Ling Kuo ${ }^{2,3}$, Shin-Cheh Chen ${ }^{2,3}$, David Chon-Fok Cheong ${ }^{1,3}$, \\ Jung-Ju Huang ${ }^{1,3,4}$ \\ ${ }^{1}$ Division of Reconstructive Microsurgery, Department of Plastic and Reconstructive Surgery and ${ }^{2}$ Department of General Surgery, Linkou \\ Chang Gung Memorial Hospital, Taoyuan; ${ }^{3}$ College of Medicine, Chang Gung University, Taoyuan; ${ }^{4}$ Center for Tissue Engineering, Linkou \\ Chang Gung Memorial Hospital, Taoyuan, Taiwan
}

Background Direct-to-implant (DTI) breast reconstruction after nipple-sparing mastectomy (NSM) with the use of acellular dermal matrix (ADM) provides reliable outcomes; however, the use of ADM is associated with a higher risk of complications. We analyzed our experiences of post-NSM DTI without ADM and identified the predictive factors of adverse surgical outcomes.

Methods Patients who underwent NSM and immediate DTI or two-stage tissue expander (TE) breast reconstruction from 2009 to 2020 were enrolled. Predictors of adverse endpoints were analyzed.

Results There were $100 \mathrm{DTI}$ and $29 \mathrm{TE}$ reconstructions. The TE group had a higher rate of postmastectomy radiotherapy ( $31 \%$ vs. $11 \% ; P=0.009)$, larger specimens $(317.37 \pm 176.42 \mathrm{~g}$ vs. $272.08 \pm 126.33 \mathrm{~g} ; \mathrm{P}=0.047)$, larger implants $(360.84 \pm 85.19 \mathrm{~g}$ vs. $298.83 \pm 81.13 \mathrm{~g} ; \mathrm{P}=0.004)$ and a higher implant/TE exposure ratio (10.3\% vs. 1\%; $P=0.035)$. In DTI reconstruction, age over 50 years (odds ratio [OR], 5.43; 95\% confidence interval $[\mathrm{Cl}], 1.50-19.74 ; \mathrm{P}=0.010$ ) and a larger mastectomy weight $(\mathrm{OR}, 1.65 ; 95 \% \mathrm{Cl}, 1.08-2.51 ; \mathrm{P}=0.021)$ were associated with a higher risk of acute complications. Intraoperative radiotherapy for the nipple-areolar complex increased the risk of acute complications $(\mathrm{OR}, 4.05 ; 95 \% \mathrm{Cl}, 1.07-15.27 ; \mathrm{P}=0.039)$ and the likelihood of revision surgery $(\mathrm{OR}, 5.57 ; 95 \% \mathrm{Cl}, 1.25-24.93 ; \mathrm{P}=0.025)$.

Conclusions Immediate DTI breast reconstruction following NSM is feasible in Asian patients with smaller breasts.

Keywords Mammaplasty / Breast implant / Mastectomy / Treatment outcomes
Correspondence: Jung-Ju Huang Division of Reconstructive Microsurgery, Department of Plastic and Reconstructive Surgery, Linkou Chang Gung Memorial Hospital, 5, Fu-Hsing St., Kweishan, Taoyuan 33305, Taiwan

Tel: +886-3-3281200 (ext. 3355)

Fax: +886-3-3972681

E-mail: jungjuhuang@gmail.com

This article was presented orally at Plastic Surgery The Meeting 2019 in San Diego, CA, USA and selected as a candidate for the best poster award at the Global Breast Cancer Conference 2019 in Seoul, Korea.

\section{INTRODUCTION}

Nipple-sparing mastectomy (NSM) has gained considerable popularity over the past decades. The oncological results of
NSM are comparable to those of skin-sparing mastectomy (SSM) in selected cases in terms of local recurrence and distant metastasis [1]. NSM is technically more challenging, with a higher complication rate than other approaches, given the addi- 
tional risk posed by poor perfusion of the nipple-areolar complex (NAC) after mastectomy [2]. As a result, breast reconstruction following NSM can be more challenging and have a higher complication rate, especially in obese patients or in those with larger breasts [3]. Nevertheless, preservation of the NAC in mastectomy with immediate breast reconstruction, either implant-based or autologous, generally results in enhanced aesthetic results and, consequently, much better quality of life $[3,4]$. This procedure is now the standard of care when patients are eligible for it.

With the application of acellular dermal matrix (ADM), implant-based breast reconstruction has primarily shifted to directto-implant (DTI) procedures $[5,6]$, especially when NSM is performed. Immediate implant-based breast reconstruction following NSM - either DTI or two-stage reconstruction with tissue expander (TE) implantation first-has been reported to have excellent aesthetic and safe oncological outcomes $[4,7]$. Nevertheless, whether DTI or two-stage reconstruction is superior remains inconclusive, with conflicting reports $[8,9]$. DTI reconstruction has been performed at our institution since the early 2000s, when ADM was not used in breast reconstruction procedures [10]. We have regularly performed DTI reconstruction after both SSM and NSM, with satisfying results in Asian patients, whose breasts are smaller than those of Western women [10]. Most of the studies discussing DTI reconstruction after NSM state that the use of ADM is required, and many of the NSMs reported in the literature were prophylactic mastectomies. Reports of clinical experiences of DTI reconstruction with NSM after breast cancer surgery without the use of ADM would be important, but are lacking.

The use of ADM in DTI breast reconstruction has been proven to lead to better overall outcomes and fewer complications than two-stage breast reconstruction. $\mathrm{ADM}$ provides adequate implant coverage in the inferior pole with improved aesthetic outcomes, better control of the inframammary fold (IMF), and less capsular contracture $[11,12]$. However, the use of $\mathrm{ADM}$ is also associated with a high number of complications, such as seroma, infection, and skin necrosis [13]. Our clinical experience of not using ADM seems to provide another option for DTI when performing breast reconstruction in Asian patients. The main purpose of this study was to present a comprehensive review of our experience and to provide a useful reference for patient selection for DTI without ADM.

\section{METHODS}

After receiving approval from the institutional review board committee (IRB Nos. $202000235 B 0$ and 202100103B0), this retrospective study recruited patients who received implantbased immediate breast reconstruction from the senior author (JJH) following NSM from October 2009 to April 2020. Patients were excluded if they had received NSM for risk-reducing prophylactic mastectomy or mastectomy to remove disseminated foreign bodies, including injected silicone and reacted granuloma, or if they were initially planned to receive autologous breast reconstruction after NSM and TE insertion. The minimal follow-up time was 3 months.

Patients were grouped into a DTI reconstruction group and a two-stage reconstruction with TE implantation first group (hereafter referred to as the TE group). The demographic and clinical data obtained included age, body mass index, smoking history, medical history (e.g., diabetes mellitus, hypertension, previous breast cancer and surgery), cancer-related characteristics (e.g., side, location, distance between the tumor and nipple, biological type of breast cancer, and TNM stage), and perioperative treatment information, including incision placement for mastectomy, axillary lymph node dissection, intraoperative radiotherapy (IORT) to the NAC, neoadjuvant and adjuvant chemotherapy, and postmastectomy radiotherapy (PMRT). Acute complications included poor wound healing, mastectomy skin flap partial necrosis, nipple necrosis, seroma, hematoma, and infection, while late complications included late wound dehiscence, infection, capsular contracture, implant/expander rupture or exposure, and implant/expander loss. We also recorded whether aesthetic revision surgery was performed for purposes including scar release, capsular contracture release, and fat grafting. Local recurrence and distant metastasis were reviewed.

\section{Surgical technique}

After the breast surgeons finished the mastectomy, the reconstruction team took over. The perfusion of the NAC and mastectomy skin flap was assessed to ensure a well-perfused breast skin envelope before implant or TE insertion; this was achieved using an intravenous injection of indocyanine green, which was visualized using a SPY device (Stryker Corp./Novadaq Technologies, Kalamazoo, MI, USA) if required (Fig. 1). If the perfusion of the skin envelope or NAC was compromised, the DTI was converted to TE insertion.

If the NAC and skin envelope were both confirmed to be well perfused, the subpectoral plane was created with careful dissection and ligation of the underlying perforators nourishing the pectoralis major (PM) muscle. The insertion of the PM muscle below the fourth intercostal level was divided. The inferior mammary fold was reinforced using 3-0 polydioxanone sutures. If the mastectomy extended beyond the anterior axillary line, two stitches were performed to recreate the lateral fold of the 

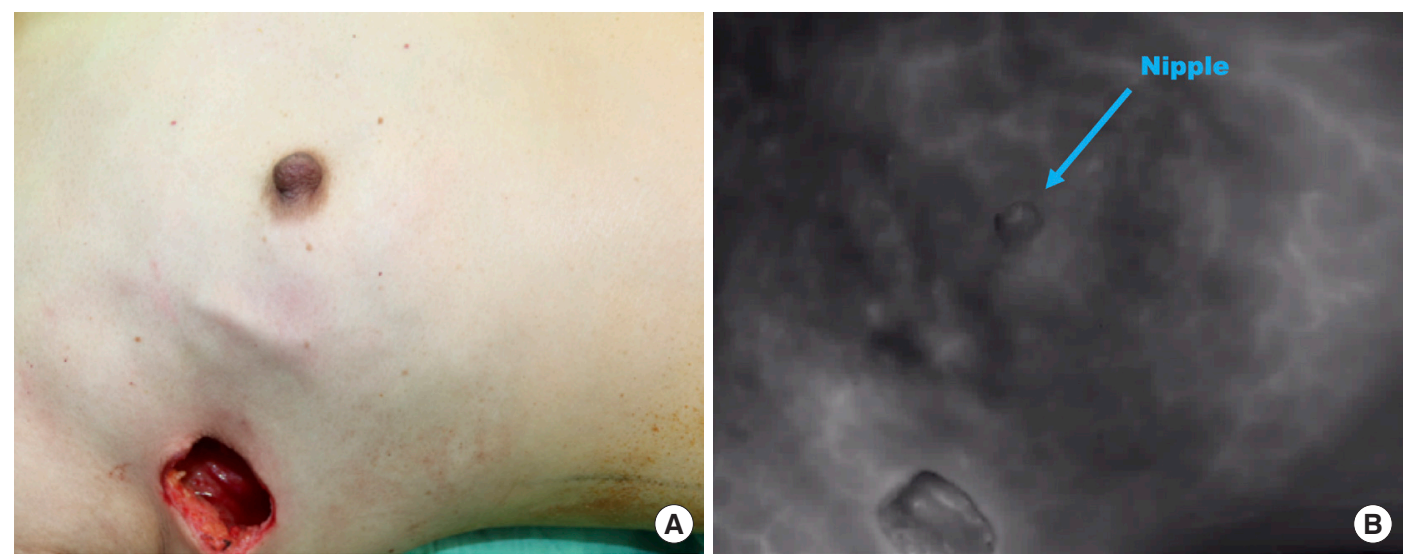

Fig. 1. Intraoperative photography grossly showing good perfusion of the preserved skin flap and the nipple in a nipple-sparing mastectomy before implant reconstruction (A), which was further confirmed by intravenous indocyanine green injection and visualized using a SPY machine (B).

breast on the anterior axillary line. A mammary sizer was first inserted to ensure adequate pocket creation and to select the implant size. After that, the pocket was irrigated with a large amount of saline, and secure hemostasis was achieved. A suction drain was inserted. Before finalizing the surgery, two stitches to anchor the inferior margin of the PM muscle to the spared skin flap were placed using Vicryl sutures to prevent muscle retraction without coverage of the implant. This helped ensure that the PM muscle would stay in the planned position and prevent the implant from sliding into the prepectoral space before complete capsule formation. The breast implant was then placed. Before wound closure, to provide better control of the lateral pocket for the breast implant, two stitches were placed to anchor the dermis of the anterior axillary line to the corresponding underlying periosteum. The stitches helped prevent lateral displacement of the implants. After wound healing, the lateral border of the reconstructed breast would then be formed. The wound was closed primarily. Patients received parenteral prophylactic antibiotics postoperatively for 3 days before being discharged.

\section{Statistical analysis}

The statistical analysis was conducted using SPSS version 23.0 (IBM Corp., Armonk, NY, USA). The chi-square or Fisher exact test was used for categorical data, while the Student $t$ test or Mann-Whitney U test was utilized for continuous data. Factors contributing to surgical outcomes were analyzed with univariable logistic regression, and factors with a P-value $<0.2$ in the univariable analysis were selected for the multivariable analysis. Backward selection was used to obtain the final models for four outcomes, in which variables with $\mathrm{P}<0.1$ were selected. A twosided $\mathrm{P}$-value $<0.05$ was considered to indicate statistical significance.
Table 1. Demographic characteristics in the direct-to-implant and tissue expander groups

\begin{tabular}{|c|c|c|c|}
\hline Characteristics & $\begin{array}{c}\text { Direct-to-implant } \\
\text { group }(n=100)\end{array}$ & $\begin{array}{l}\text { Tissue expander } \\
\text { group }(\mathrm{n}=29)\end{array}$ & P-value \\
\hline Age (yr) & $44.17 \pm 7.03$ & $40.97 \pm 8.70$ & $0.043^{\mathrm{a})}$ \\
\hline Body mass index $\left(\mathrm{kg} / \mathrm{m}^{2}\right)$ & $21.15 \pm 2.54$ & $22.91 \pm 4.07$ & $0.025^{\mathrm{a})}$ \\
\hline History of smoking & $2(2)$ & 0 & 1.000 \\
\hline \multicolumn{4}{|l|}{ Medical history } \\
\hline Diabetes mellitus & $1(1)$ & $2(6.9)$ & 0.127 \\
\hline Hypertension & $2(2)$ & 0 & 1.000 \\
\hline Previous breast cancer & $8(8)$ & $2(6.9)$ & 1.000 \\
\hline Previous mastectomy & $13(13)$ & $3(10.3)$ & 1.000 \\
\hline Previous lymph node dissection & $5(5)$ & 0 & 0.584 \\
\hline Hospital stay (day) & $7.78 \pm 2.46$ & $7.17 \pm 2.36$ & 0.206 \\
\hline Follow-up time (mo) & $20.11 \pm 23.22$ & $14.61 \pm 10.57$ & 0.540 \\
\hline
\end{tabular}

Values are presented as mean $\pm \mathrm{SD}$ or number (\%).

${ }^{\text {a) }}$ Statistically significant, $\mathrm{P}<0.05$.

\section{RESULTS}

A total of 129 breasts in 123 patients undergoing immediate breast reconstruction following NSM were enrolled, including 100 and 29 cases in the DTI and TE groups, respectively. Table 1 lists their demographic characteristics. The patients in the TE group were younger ( $40.97 \pm 8.70$ years vs. $44.17 \pm 7.03$ years; $\mathrm{P}=0.043)$ and had a higher average body mass index (22.91 \pm $4.07 \mathrm{~kg} / \mathrm{m}^{2}$ vs. $\left.21.15 \pm 2.54 \mathrm{~kg} / \mathrm{m}^{2} ; \mathrm{P}=0.025\right)$ than those in the DTI group (Table 1). The breast cancer characteristics and treatments are shown in Table 2; no significance between-group differences were found in the biological type of the cancer, the cancer stage, nodal surgery, neoadjuvant/adjuvant chemotherapy, or hormone therapy. More patients received PMRT in the TE group than in the DTI group (31\% and $11 \%$, respectively; $\mathrm{P}=0.009$ ).

The postoperative outcomes are summarized in Table 3 . The 
Table 2. Tumor characteristics and associated treatment between the direct-to-implant and tissue expander groups

\begin{tabular}{|c|c|c|c|}
\hline Characteristics & $\begin{array}{c}\text { Direct-to-implant } \\
\text { group }(n=100)\end{array}$ & $\begin{array}{l}\text { Tissue expander } \\
\text { group }(n=29)\end{array}$ & P-value \\
\hline Side & & & 0.593 \\
\hline Left & $47(47)$ & $12(41.4)$ & \\
\hline Right & $53(53)$ & $17(58.6)$ & \\
\hline Location & & & 0.404 \\
\hline UOQ & $52(52)$ & $14(48.3)$ & \\
\hline LOQ & $17(17)$ & $2(6.9)$ & \\
\hline UIQ & $20(20)$ & $8(27.6)$ & \\
\hline LIQ & $11(11)$ & $5(17.2)$ & \\
\hline Distance from the nipple $(\mathrm{cm})$ & $2.83 \pm 1.28$ & $2.66 \pm 2.16$ & 0.195 \\
\hline Histological type & & & 0.174 \\
\hline Ductal carcinoma in situ & $30(30)$ & $5(17.2)$ & \\
\hline Invasive carcinoma & $70(70)$ & $24(82.8)$ & \\
\hline T stage & & & 0.322 \\
\hline Tis & $20(20)$ & $5(17.2)$ & \\
\hline $\mathrm{T} 1$ & $50(50)$ & $11(37.9)$ & \\
\hline$\geq \mathrm{T} 2$ & $30(30)$ & $13(44.9)$ & \\
\hline N stage & & & 0.779 \\
\hline NO & $75(75)$ & $21(72.4)$ & \\
\hline$\geq \mathrm{N} 1$ & $25(25)$ & $8(27.6)$ & \\
\hline M stage & & & 0.400 \\
\hline MO & 99 (99) & 28 (96.9) & \\
\hline M1 & $1(1)$ & $1(3.10)$ & \\
\hline \multicolumn{4}{|l|}{ IHC type } \\
\hline $\mathrm{HR}+$ & $79(79)$ & 24 (82.8) & 0.657 \\
\hline HER2+ & $29(29)$ & $10(34.5)$ & 0.571 \\
\hline Axillary lymph node dissection & $20(20)$ & $9(31.0)$ & 0.210 \\
\hline IORT targeting the NAC & $18(18)$ & $3(10.3)$ & 0.404 \\
\hline Neoadjuvant chemotherapy & $15(15)$ & $8(27.6)$ & 0.119 \\
\hline Adjuvant chemotherapy & $48(48)$ & $16(55.2)$ & 0.496 \\
\hline Hormone therapy & $55(55)$ & $18(62)$ & 0.499 \\
\hline PMRT & $11(11)$ & $9(31.0)$ & $0.009^{a)}$ \\
\hline Contralateral augmentation & $14(14)$ & $2(6.9)$ & 0.522 \\
\hline
\end{tabular}

Values are presented as number (\%) or mean \pm SD.

$U O Q$, upper-outer quadrant; LOQ, lower-outer quadrant; UIQ, upper-inner quadrant; LIQ, lower-inner quadrant; IHC, immunohistochemistry; HR, hormone receptor; HER2, human epidermal growth factor receptor 2; IORT, intraoperative radiotherapy; NAC, nipple-areolar complex; PMRT, postmastectomy radiotherapy.

a) Statistically significant, $\mathrm{P}<0.05$.

TE group had larger mastectomy specimens $(317.37 \pm 176.42 \mathrm{~g}$ and $272.08 \pm 126.33 \mathrm{~g}$, respectively; $\mathrm{P}=0.047)$ and larger implants ( $360.84 \pm 85.19 \mathrm{~g}$ and $298.83 \pm 81.13 \mathrm{~g}$, respectively; $\mathrm{P}=$ $0.004)$. Acute and late complications were similar between the groups, except that late implant/expander exposure was significantly more frequent in the TE group (10.3\%) than in the DTI group (1\%) $(\mathrm{P}=0.035)$. Six of the $29(20.7 \%)$ patients in the TE groups were converted to free flap reconstruction due to the patient's preference after TE insertion.

To identify factors contributing to acute and late complications, univariate logistic regression was conducted for overall complications as a whole and for implant removal and aesthetic revision surgery in particular, as shown in Tables 4 and 5. Age
Table 3. Intraoperative data and postoperative outcomes between the direct-to-implant and tissue expander groups

\begin{tabular}{|c|c|c|c|}
\hline Characteristics & $\begin{array}{l}\text { Direct-to-implant } \\
\text { group }(n=100)\end{array}$ & $\begin{array}{l}\text { Tissue expander } \\
\text { group }(n=29)\end{array}$ & P-value \\
\hline \multicolumn{3}{|c|}{ Incision placement of mastectomy } & 0.570 \\
\hline Inframammary fold & $9(9)$ & $3(10.3)$ & \\
\hline Periareolar & $11(11)$ & $6(20.7)$ & \\
\hline Lateral & $57(57)$ & $14(48.3)$ & \\
\hline Other incisions & $23(23)$ & $6(20.7)$ & \\
\hline Specimen size (g) & $272.08 \pm 126.33$ & $317.37 \pm 176.42$ & $0.047^{c)}$ \\
\hline Implant size (g) & $298.83 \pm 81.13$ & $360.84 \pm 85.19$ & $0.004^{c)}$ \\
\hline \multicolumn{4}{|l|}{ Implant type $e^{a)}$} \\
\hline Silicone & $95(95)$ & $18(100)$ & 1.000 \\
\hline Saline & $5(5)$ & 0 & \\
\hline Implant surface ${ }^{\text {a) }}$ & & & 0.447 \\
\hline Texture & $40(40)$ & $9(50.0)$ & \\
\hline Smooth & $60(60)$ & $9(50.0)$ & \\
\hline \multicolumn{4}{|l|}{ Acute complications } \\
\hline Overall & $19(19)$ & $8(23.1)$ & 0.317 \\
\hline Poor wound healing & $6(6)$ & $3(10.3)$ & 0.420 \\
\hline Skin flap necrosis & $8(8)$ & $6(20.7)$ & 0.053 \\
\hline Nipple necrosis & $3(3)$ & $2(6.9)$ & 0.313 \\
\hline Seroma & $1(1)$ & 0 & 1.000 \\
\hline Hematoma & $1(1)$ & 0 & 1.000 \\
\hline Infection & $4(4)$ & 0 & 0.574 \\
\hline \multicolumn{4}{|l|}{ Late complications } \\
\hline Overall & $21(21)$ & $8(27.6)$ & 0.454 \\
\hline Capsular contracture & $9(9)$ & $1(3.4)$ & 0.455 \\
\hline Implant/expander rupture & $4(4)$ & 0 & 0.574 \\
\hline Implant/expander exposure & $1(1)$ & $3(10.3)$ & $0.035^{c)}$ \\
\hline Wound dehiscence & $8(8)$ & $5(17.2)$ & 0.146 \\
\hline Infection & $6(6)$ & $4(13.8)$ & 0.231 \\
\hline \multicolumn{4}{|l|}{ Oncologic outcome } \\
\hline Local recurrence & $1(1.1)$ & $2(7.7)$ & 0.127 \\
\hline Distant metastasis & $5(5)$ & $1(3.4)$ & 1.000 \\
\hline $\begin{array}{l}\text { Implant/tissue expander } \\
\text { removal }\end{array}$ & $6(6)$ & $5(17.2)$ & 0.069 \\
\hline Conversion to free flap b) & 0 & $6(20.7)$ & $<0.001^{c)}$ \\
\hline \multicolumn{4}{|l|}{ Revision aesthetic surgery } \\
\hline Overall & $8(8)$ & 0 & 0.197 \\
\hline Scar release & $4(4)$ & $1(3.4)$ & 1.000 \\
\hline Fat graft & $3(3)$ & 0 & 1.000 \\
\hline Skin revision & $4(4)$ & 0 & 0.574 \\
\hline
\end{tabular}

Values are presented as number (\%) or mean \pm SD.

a)Among 29 patients with tissue expander, 6 converted to a flap, 4 experienced expander loss, and 1 experienced implant loss; ${ }^{b}$ Conversion to autologous reconstruction without complications; ') Statistically significant, $\mathrm{P}<0.05$.

older than 50 years (odds ratio [OR], 4.63; 95\% confidence interval $[\mathrm{CI}], 1.52-14.05 ; \mathrm{P}=0.007)$ and a larger mastectomy weight (per $100 \mathrm{~g})(\mathrm{OR}, 1.80$; 95\% CI, 1.20-2.68; $\mathrm{P}=0.004)$ were associated with a higher risk of overall acute complications. IORT targeting the NAC (OR, 5.57; 95\% CI, 1.25-24.93; P= $0.025)$ increased the risk of revision surgery. Multivariable logistic regression was further conducted for variables with $\mathrm{P}<0.1$ in the univariate model. Consistent with the univariate logistic re- 
Table 4. Univariable logistic regression for complications in the direct-to-implant group

\begin{tabular}{|c|c|c|c|c|}
\hline \multirow{2}{*}{ Variable } & \multicolumn{2}{|c|}{ Acute complications } & \multicolumn{2}{|c|}{ Late complications } \\
\hline & OR $(95 \% \mathrm{Cl})$ & P-value & $\mathrm{OR}(95 \% \mathrm{Cl})$ & P-value \\
\hline \multicolumn{5}{|l|}{ Age (yr) } \\
\hline$<50$ & Reference & & Reference & \\
\hline$\geq 50$ & $4.63(1.52-14.05)$ & $0.007^{\mathrm{b})}$ & $2.03(0.66-6.21)$ & 0.214 \\
\hline \multicolumn{5}{|l|}{$\mathrm{BMI}\left(\mathrm{kg} / \mathrm{m}^{2}\right)$} \\
\hline$<25$ & Reference & & Reference & \\
\hline$\geq 25$ & $1.07(0.11-10.15)$ & 0.953 & $0.938(0.10-8.86)$ & 0.955 \\
\hline History of smoking & - & - & - & - \\
\hline \multicolumn{5}{|l|}{ Medical history } \\
\hline Diabetes mellitus & - & - & - & - \\
\hline Hypertension & $4.44(0.27-74.46)$ & 0.300 & - & - \\
\hline Previous breast cancer & $0.59(0.07-5.08)$ & 0.629 & $0.51(0.06-4.43)$ & 0.545 \\
\hline Previous mastectomy & $2.13(0.58-7.85)$ & 0.254 & $1.15(0.29-4.62)$ & 0.844 \\
\hline Previous lymph node dissection & $1.07(0.11-10.15)$ & 0.953 & $0.94(0.10-8.86)$ & 0.955 \\
\hline \multicolumn{5}{|l|}{ Tumor side } \\
\hline Left & Reference & & Reference & \\
\hline Right & $1.67(0.60-4.68)$ & 0.327 & $0.60(0.23-1.58)$ & 0.297 \\
\hline \multicolumn{5}{|l|}{ Tumor location } \\
\hline UOQ & Reference & & Reference & \\
\hline LOQ & $1.69(0.44-6.53)$ & 0.445 & $0.64(0.16-2.60)$ & 0.535 \\
\hline$U I Q$ & $1.83(0.52-6.47)$ & 0.346 & $0.53(0.13-2.10)$ & 0.366 \\
\hline LIQ & $1.22(0.22-6.74)$ & 0.818 & $0.67(0.13-3.49)$ & 0.631 \\
\hline Tumor distance from the nipple & $1.14(0.78-1.67)$ & 0.507 & $1.10(0.76-1.60)$ & 0.607 \\
\hline \multicolumn{5}{|l|}{ Tumor histological type } \\
\hline Ductal carcinoma in situ & Reference & & Reference & \\
\hline Invasive carcinoma & $1.25(0.41-3.85)$ & 0.697 & $0.63(0.23-1.72)$ & 0.365 \\
\hline \multicolumn{5}{|l|}{ T stage } \\
\hline Tis & Reference & & Reference & \\
\hline $\mathrm{T} 1$ & $0.92(0.21-3.99)$ & 0.914 & $0.58(0.18-1.90)$ & 0.371 \\
\hline$\geq \mathrm{T} 2$ & $2.43(0.57-10.40)$ & 0.232 & $0.47(0.12-1.81)$ & 0.270 \\
\hline \multicolumn{5}{|l|}{ N stage } \\
\hline NO & Reference & & Reference & \\
\hline$\geq \mathrm{N} 1$ & $1.51(0.50-4.50)$ & 0.464 & $0.92(0.30-2.84)$ & 0.887 \\
\hline \multicolumn{5}{|l|}{ M stage } \\
\hline M0 & Reference & & Reference & \\
\hline M1 & - & & - & \\
\hline \multicolumn{5}{|l|}{ Tumor IHC type } \\
\hline $\mathrm{HR}+$ & $2.61(0.55-12.31)$ & 0.227 & $0.81(0.26-2.55)$ & 0.722 \\
\hline HER2+ & $0.40(0.11-1.48)$ & 0.169 & $1.70(0.62-4.68)$ & 0.304 \\
\hline Axillary lymph node dissection & $1.57(0.49-5.04)$ & 0.447 & $0.61(0.16-2.31)$ & 0.465 \\
\hline IORT targeting the NAC & $2.65(0.84-8.34)$ & 0.095 & $2.23(0.72-6.90)$ & 0.163 \\
\hline Neoadjuvant chemotherapy & $1.08(0.27-4.27)$ & 0.915 & $0.23(0.03-1.88)$ & 0.171 \\
\hline Adjuvant chemotherapy & $2.14(0.77-6.00)$ & 0.147 & $0.35(0.12-1.00)$ & 0.050 \\
\hline PMRT & $0.39(0.05-3.29)$ & 0.390 & $0.35(0.04-2.86)$ & 0.324 \\
\hline \multicolumn{5}{|l|}{ Incision placement of mastectomy } \\
\hline IMF & Reference & & Reference & \\
\hline Periareolar & $1.14(0.18-7.28)$ & 0.888 & $0.20(0.02-2.39)$ & 0.203 \\
\hline Lateral & $0.28(0.06-1.38)$ & 0.118 & $0.43(0.09-2.00)$ & 0.278 \\
\hline Other incisions & $0.56(0.10-3.05)$ & 0.499 & $0.88(0.17-4.54)$ & 0.874 \\
\hline Specimen size $(100 \mathrm{~g})^{\mathrm{a})}$ & $1.80(1.20-2.68)$ & $0.004^{b)}$ & $1.09(0.75-1.58)$ & 0.660 \\
\hline Specimen size-implant size $(100 \mathrm{~g})^{\mathrm{a}}$ & $1.46(0.88-2.41)$ & 0.142 & $1.55(0.86-2.78)$ & 0.146 \\
\hline
\end{tabular}

$\mathrm{OR}$, odds ratio; $\mathrm{Cl}$, confidence interval; BMI, body mass index; UOQ, upper-outer quadrant; LOQ, lower-outer quadrant; UIQ, upper-inner quadrant; LIQ, lower-inner quadrant; IHC, immunohistochemistry; HR, hormone receptor; HER2, human epidermal growth factor receptor 2; IORT, intraoperative radiotherapy; NAC, nipple-areolar complex; PMRT, postmastectomy radiotherapy; IMF, inframammary fold.

${ }^{\text {a)}}$ OR calculated for a 100-g weight difference; b)Statistically significant, $P<0.05$. 
Table 5. Univariable logistic regression for the need for secondary surgery in the direct-to-implant group

\begin{tabular}{|c|c|c|c|c|}
\hline \multirow{2}{*}{ Variable } & \multicolumn{2}{|c|}{ Implant removal } & \multicolumn{2}{|c|}{ Revision aesthetic surgery } \\
\hline & $\mathrm{OR}(95 \% \mathrm{Cl})$ & P-value & $\mathrm{OR}(95 \% \mathrm{Cl})$ & P-value \\
\hline \multicolumn{5}{|l|}{ Age (yr) } \\
\hline$<50$ & Reference & & Reference & \\
\hline$\geq 50$ & $2.27(0.38-13.39)$ & 0.367 & $1.47(0.27-7.93)$ & 0.654 \\
\hline \multicolumn{5}{|l|}{$\mathrm{BMI}\left(\mathrm{kg} / \mathrm{m}^{2}\right)$} \\
\hline$<25$ & Reference & & Reference & \\
\hline$\geq 25$ & - & - & - & - \\
\hline History of smoking & - & - & - & - \\
\hline \multicolumn{5}{|l|}{ Medical history } \\
\hline Diabetes mellitus & - & - & - & - \\
\hline Hypertension & - & - & - & - \\
\hline Previous breast cancer & $2.49(0.25-24.33)$ & 0.434 & - & - \\
\hline Previous mastectomy & $1.04(0.12-9.43)$ & 0.971 & $0.95(0.11-8.44)$ & 0.965 \\
\hline Previous lymph node dissection & $4.50(0.42-48.10)$ & 0.213 & - & - \\
\hline \multicolumn{5}{|l|}{ Tumor side } \\
\hline Left & Reference & & Reference & \\
\hline Right & $0.88(0.17-4.59)$ & 0.879 & $0.88(0.21-3.72)$ & 0.859 \\
\hline \multicolumn{5}{|l|}{ Tumor location } \\
\hline UOQ & Reference & & Reference & \\
\hline LOQ & $3.33(0.43-25.72)$ & 0.248 & $1.02(0.10-10.52)$ & 0.986 \\
\hline UIQ & $1.32(0.11-15.37)$ & 0.827 & $1.82(0.28-11.76)$ & 0.532 \\
\hline LIQ & $2.50(0.21-30.29)$ & 0.472 & $3.63(0.53-24.89)$ & 0.189 \\
\hline Tumor distance from the nipple & $1.23(0.67-2.26)$ & 0.500 & $1.40(0.83-2.37)$ & 0.211 \\
\hline \multicolumn{5}{|l|}{ Tumor histological type } \\
\hline Ductal carcinoma in situ & Reference & & Reference & \\
\hline Invasive carcinoma & $2.23(0.25-19.96)$ & 0.473 & $1.31(0.25-6.91)$ & 0.748 \\
\hline \multicolumn{5}{|l|}{ T stage } \\
\hline Tis & Reference & & Reference & \\
\hline $\mathrm{T} 1$ & $0.39(0.02-6.52)$ & 0.511 & - & - \\
\hline$\geq \mathrm{T} 2$ & $2.92(0.30-28.29)$ & 0.354 & - & - \\
\hline \multicolumn{5}{|l|}{$\mathrm{N}$ stage } \\
\hline NO & Reference & & Reference & \\
\hline$\geq \mathrm{N} 1$ & $1.54(0.27-8.98)$ & 0.629 & $0.41(0.05-3.46)$ & 0.409 \\
\hline \multicolumn{5}{|l|}{ M stage } \\
\hline MO & Reference & & Reference & \\
\hline M1 & - & - & - & - \\
\hline \multicolumn{5}{|l|}{ Tumor IHC type } \\
\hline $\mathrm{HR}+$ & $1.35(0.15-12.23)$ & 0.789 & $0.41(0.09-1.86)$ & 0.245 \\
\hline HER2+ & $0.47(0.05-4.22)$ & 0.501 & $0.56(0.12-2.51)$ & 0.445 \\
\hline Axillary lymph node dissection & $0.79(0.09-7.16)$ & 0.834 & $0.55(0.06-4.74)$ & 0.585 \\
\hline IORT targeting the NAC & $2.44(0.41-14.46)$ & 0.327 & $5.57(1.25-24.93)$ & $0.025^{\text {b) }}$ \\
\hline Neoadjuvant chemotherapy & - & - & - & - \\
\hline Adjuvant chemotherapy & $2.27(0.40-13.01)$ & 0.356 & $1.09(0.26-4.63)$ & 0.906 \\
\hline PMRT & $1.68(0.18-18.86)$ & 0.651 & - & - \\
\hline \multicolumn{5}{|l|}{ Incision placement for mastectomy } \\
\hline IMF & Reference & & Reference & \\
\hline Periareolar & - & & - & \\
\hline Lateral & $0.13(0.02-1.05)$ & 0.056 & $0.60(0.06-6.11)$ & 0.669 \\
\hline Other incisions & $0.33(0.04-2.83)$ & 0.314 & $1.20(0.11-13.32)$ & 0.882 \\
\hline Specimen size $(100 \mathrm{~g})^{\mathrm{a})}$ & $1.07(0.57-2.02)$ & 0.826 & $1.40(0.85-2.32)$ & 0.188 \\
\hline Specimen size-implant size $(100 \mathrm{~g})^{\mathrm{a}}$ & $0.95(0.45-2.01)$ & 0.888 & $1.28(0.64-2.58)$ & 0.491 \\
\hline
\end{tabular}

$\mathrm{OR}$, odds ratio; $\mathrm{Cl}$, confidence interval; BMI, body mass index; UOQ, upper-outer quadrant; LOQ, lower-outer quadrant; UIQ, upper-inner quadrant; LIQ, lower-inner quadrant; IHC, immunohistochemistry; HR, hormone receptor; HER2, human epidermal growth factor receptor 2; IORT, intraoperative radiotherapy; NAC, nipple-areolar complex; PMRT, postmastectomy radiotherapy; IMF, inframammary fold.

a)OR calculated for a 100-g weight difference; b) Statistically significant, $P<0.05$. 
gression, age greater than 50 years (OR, 5.43; 95\% CI, 1.5019.74; $\mathrm{P}=0.010$ ) and a larger mastectomy weight (per $100 \mathrm{~g}$ ) (OR, 1.65; 95\% CI, 1.08-2.51; $\mathrm{P}=0.021$ ) were associated with a higher risk of acute complications. IORT targeting the NAC contributed to both a higher risk of acute complications (OR, 4.05; 95\% CI, 1.07-15.27; P = 0.039) and to a higher likelihood of requiring aesthetic revision surgery $(\mathrm{OR}, 5.57 ; 95 \% \mathrm{CI}$, 1.25-24.93; $\mathrm{P}=0.025)$. Although advanced age, mastectomy weight, and IORT targeting the NAC were found to be associated with a higher overall complication rate and a higher likelihood of requiring revision surgery, neither factor was associated with any specific complication when the complications were analyzed individually (Table 6).

\section{DISCUSSION}

Our findings supported the hypothesis that, with proper patient selection, DTI reconstruction can be successfully performed after NSM for breast cancer treatment in Asian patients with a low body mass index and small to moderate-sized breasts, even without the use of $\mathrm{ADM}$ (Figs. 2, 3).

Immediate DTI breast reconstruction was first introduced in

Table 6. Multivariable logistic regression for adverse outcomes in the direct-to-implant group

\begin{tabular}{|c|c|c|c|c|c|c|c|c|}
\hline \multirow{2}{*}{ Variable } & \multicolumn{2}{|c|}{ Acute complications } & \multicolumn{2}{|c|}{ Late complications } & \multicolumn{2}{|c|}{ Implant removal } & \multicolumn{2}{|c|}{ Revision aesthetic surgery } \\
\hline & $\mathrm{OR}(95 \% \mathrm{Cl})$ & P-value & $\mathrm{OR}(95 \% \mathrm{Cl})$ & P-value & $\mathrm{OR}(95 \% \mathrm{Cl})$ & P-value & $\mathrm{OR}(95 \% \mathrm{Cl})$ & P-value \\
\hline Age $\geq 50 \mathrm{yr}$ & $5.43(1.50-19.74)$ & 0.010 & - & - & - & - & - & - \\
\hline IORT targeting the NAC & $4.05(1.07-15.27)$ & 0.039 & - & - & - & - & $5.57(1.25-24.93)$ & 0.025 \\
\hline Adjuvant chemotherapy & - & - & NS & NS & - & - & - & - \\
\hline Incision placement for mastectomy & - & - & - & - & NS & NS & - & - \\
\hline Lateral & - & - & - & - & - & - & - & - \\
\hline Specimen size $(100 \mathrm{~g})^{\mathrm{a})}$ & $1.65(1.08-2.51)$ & 0.021 & - & - & - & - & - & - \\
\hline
\end{tabular}

$\mathrm{OR}$, odds ratio; $\mathrm{Cl}$, confidence interval; IORT, intraoperative radiotherapy; NAC, nipple-areolar complex; NS, not significant.

a) $O R$ calculated for a 100-g weight difference.
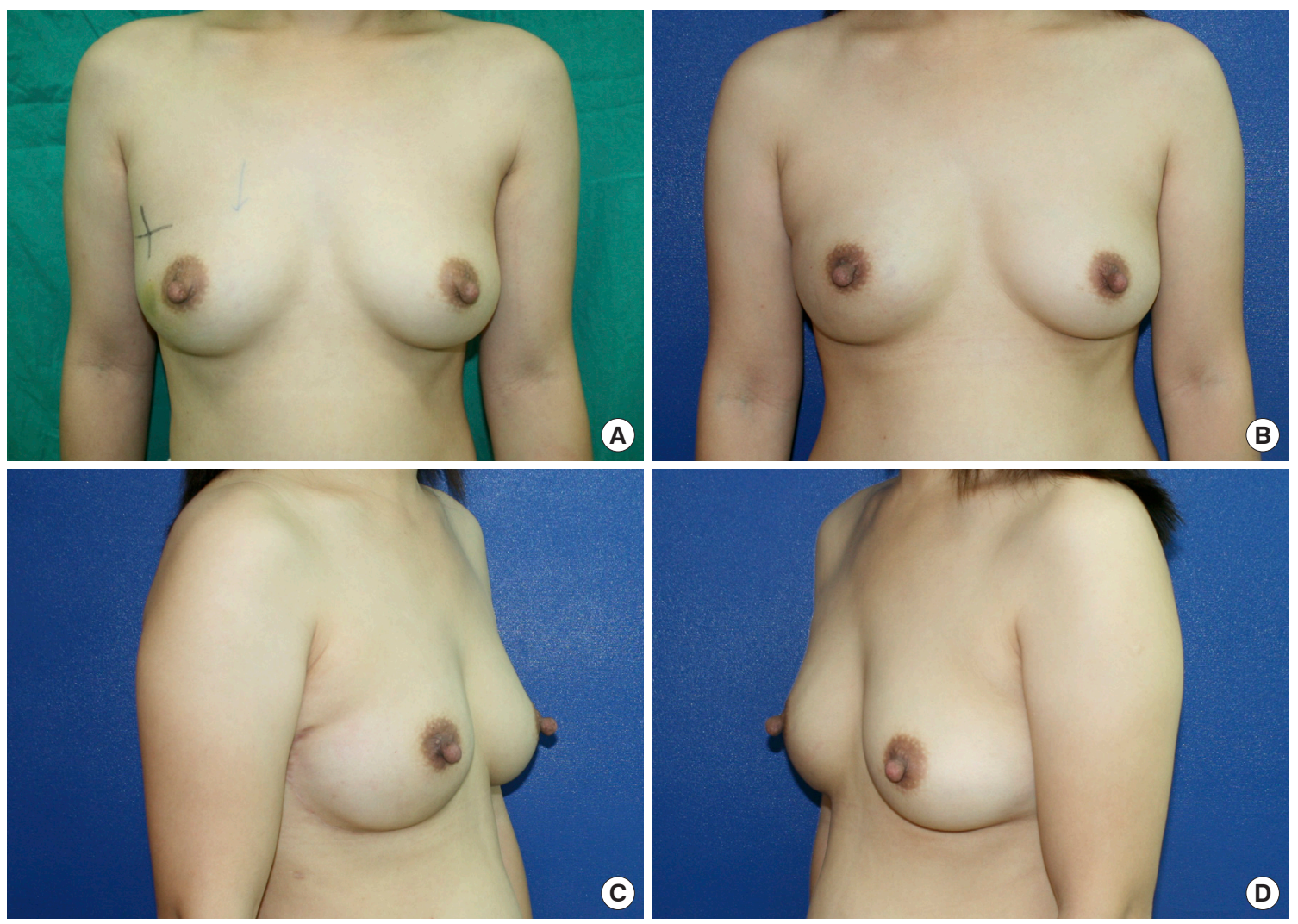

Fig. 2. Photographs of a 39-year-old patient who underwent right-side direct-to-implant breast reconstruction following nipple-sparing mastectomy for ductal carcinoma in situ without the use of acellular dermal matrix. (A) Preoperative anterior view. Postoperative anterior view (B), right lateral view (C), and left lateral view (D). Postoperative photos were taken at the 5-month follow-up. 

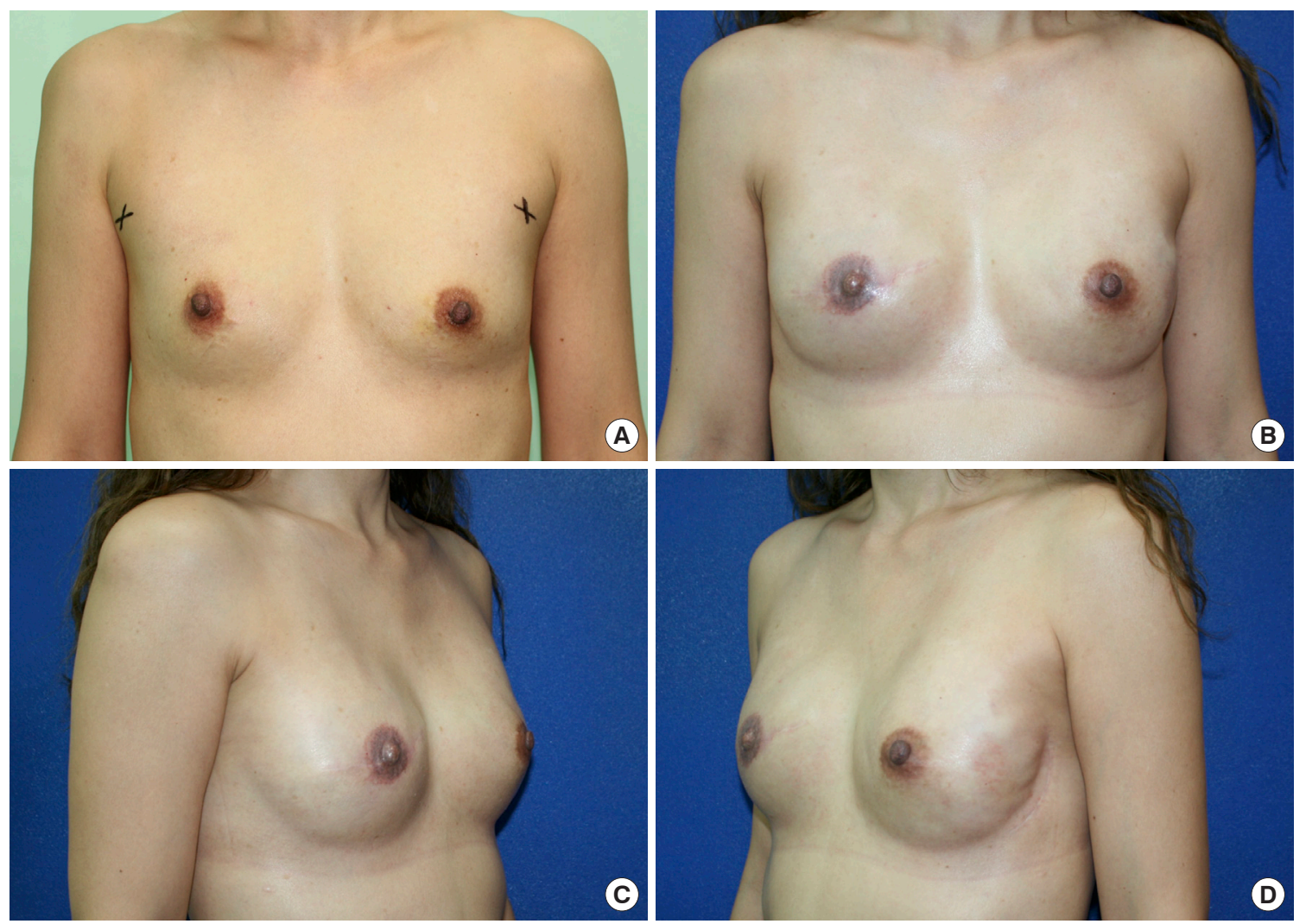

Fig. 3. A 47-year-old female patient with bilateral breast cancer, staged T1 miNo on the right side and atypical ductal hyperplasia on the left side. She received double mastectomy, with skin-sparing mastectomy on the right side and nipple-sparing mastectomy on the left side. Simultaneous bilateral direct-to-implant reconstruction was performed immediately. (A) Preoperative anterior view. Postoperative anterior (B), right lateral (C), and left lateral (D) views after right-side nipple and areolar reconstruction at the 12-month follow-up.

the early 2000s and popularized after ADM was applied for implant-based reconstruction $[10,14]$. However, conflicting results have been reported, and the preference for DTI reconstruction over two-stage implant-based reconstruction seems to be highly reliant on the use of ADM [8,11,15-20]. As presented herein, we found that the frequency of implant/TE exposure was significantly lower in the DTI group (1\%) than in the TE group $(12.3 \%, \mathrm{P}=0.032)$ (Table 3$)$. However, selection bias may have affected these findings, since two-stage reconstruction has long been suggested if PMRT is required, and we tend to perform two-stage reconstruction if PMRT is required or if the perfusion of the mastectomy skin flap is compromised.

Implant loss was observed in both groups for different reasons. In the DTI group, six cases of late implant loss were encountered, with two due to capsular contracture and removal at the patient's request, three due to infection, and one due to delayed repeated wound dehiscence after radiotherapy. In the TE group, TE/implant loss occurred in five patients: one who developed capsular contracture and requested removal, three who experienced repeated wound disruption with TE/implant exposure, and one who developed breast cellulitis. Six patients decided to convert from implant-based reconstruction to autologous reconstruction.

Despite the advantages of DTI reconstruction, several factors increased the risk of complications, including age over 50 years, a larger mastectomy specimen, and delivery of IORT targeting the NAC. The size of the mastectomy has been reported as a factor contributing to adverse outcomes in DTI breast reconstruction by various authors $[17,18,21]$. In our study, the size of the mastectomy specimen was also found to be a significant contributing risk factor for acute complications of DTI reconstruction (OR, 1.65; 95\% CI, 1.08-2.51; P=0.021). Interestingly, while mastectomy size negatively impacted the surgical outcomes, the same finding did not apply to the size of the breast implants (Table 4). Similar findings were presented by Negenborn et al. [21], who showed that the complication rate was associated with the size of the mastectomy specimen, but not the size of the inserted breast implant. A possible reason for this is that the implants used for reconstruction tend to be smaller than the mastectomy specimens, and therefore were not significantly different from one another.

In addition, age greater than 50 years contributed to a higher 
acute complication rate (OR, 5.43; 95\% CI, 1.50-19.74; $\mathrm{P}=$ 0.010 ), which has also been reported as a risk factor by Hunsicker et al. [22]. Larger breasts and the breasts of women of more advanced age are associated with ptosis, and the collateral circulation from the surrounding normal tissue to the breast envelope of ptotic breasts is relatively more restricted, resulting in more frequent skin necrosis and a larger dead space for hematoma and seroma accumulation.

IORT targeting the NAC during NSM has been reported to improve local cancer control and radiation safety [23]. Our approach includes an INTRABEAM IORT device (Zeiss Meditech, Jena, Germany) with a single dose of 6-12 Gy to irradiate the NAC, followed by immediate breast reconstruction. IORT targeting the NAC was associated with a higher risk of acute complications in our small series (OR, 4.05; 95\% CI, 1.0715.27; $\mathrm{P}=0.039)$ and a higher frequency of aesthetic revision surgery (OR, 5.57; 95\% CI, 1.25-24.93; P=0.025). There was a higher likelihood of requiring revision surgery, including nipple revision, nipple reconstruction, and scar revision on the incision site, since IORT targeting the NAC obstructs the collateral circulation through the NAC to the incision site. The risks of aesthetic complications have rarely been discussed in the literature. Both our univariate and multivariate logistic regression analyses identified IORT as a contributing factor to acute complications and the need for secondary aesthetic touchup.

Unlike previous publications [24], in our series, incision placement had no significant relationship with adverse surgical outcomes. In previous studies regarding various incision patterns, making the incision through the IMF yielded more favorable outcomes [25]. We have relatively little experience with the IMF approach (9\%), whereas a lateral incision (57\%) was the most widely used approach due to aesthetic considerations and the possible need for future axillary nodal surgery.

The application of $\mathrm{ADM}$ in implant-based reconstruction has increased in the last 10 years $[11,26]$. However, the use of ADM increases medical costs, and $\mathrm{ADM}$ implantation also inevitably results in a higher risk of some complications, such as infection or seroma formation $[13,22,26,27]$. To balance the costs, reduce the complications, and enhance the aesthetic results, it is important to select candidates who would benefit more from ADM or no ADM in DTI reconstruction. The application of ADM has been recommended in DTI breast reconstruction and it is considered helpful when larger breast implants are required $[11,26,28,29]$. In the current study, we successfully performed DTI reconstruction after NSM without ADM. Although the implants did not have support in the inferior pole, we did not experience bottom-up or implant migration from the subpectoral to the prepectoral plane. These favorable outcomes were be- lieved to be related to the surgical techniques used to fix the PM muscle to the skin flap and the smaller implants required in our population. Breast size represents a significant difference between Asian and Western populations; consequently, the breast implants used for breast reconstruction in our series were much smaller than those reported in most previous studies. Ninetythree of the 100 mastectomies used implants that were smaller than $500 \mathrm{~g}$, and the breast implants used in our patient cohort were generally small $(298.83 \pm 81.13 \mathrm{~mL}$ on average). Our results suggest that post-NSM DTI reconstruction without ADM application is feasible, with reasonable complication rates and satisfying aesthetic results, when the reconstructed breasts are small and non-ptotic.

Despite the satisfactory results, there are a few limitations of our study. First, a control group of patients who underwent DTI reconstructions with $\mathrm{ADM}$ was lacking. However, this fact reflects our early start and consistent performance of DTI without the use of ADM. Second, the outcomes demonstrated in our study were all on the surgical side; patient-reported outcomes will be obtained later to confirm these results. Third, the number of patients remains small, and a larger-scale study needs to be carried out in the future. Fourth, the follow-up duration varied, with the shortest period being only 3 months. A study with a longer follow-up time will be continued to evaluate long-term outcomes. Finally, the choice between DTI and TE was not randomly controlled, and we tended to perform DTI if possible. Only patients who had poorly perfused skin envelopes, inadequate skin envelopes, or were known to need PMRT received TE insertion after mastectomy. This might have contributed to their higher frequency of complications, particularly skin flap necrosis and wound healing problems. Selection bias might have contributed to the higher complication rate in the TE group.

Although more complications tended to occur in patients with larger breasts and those over 50 years old, DTI breast reconstruction after NSM remains a feasible and safe procedure with reasonable overall acute and late complications in highly selected Asian patients.

\section{NOTES}

\section{Conflict of interest}

Jung-Ju Huang is an editorial board member of the journal but was not involved in the peer reviewer selection, evaluation, or decision process of this article. No other potential conflicts of interest relevant to this article were reported. 


\section{Ethical approval}

The study was approved by the Institutional Review Board of Chang Gung Medical Foundation (IRB Nos. 202000-235B0 and 202100103B0) and performed in accordance with the principles of the Declaration of Helsinki. Written informed consent was obtained.

\section{Patient consent}

The patients provided written informed consent for the publication and the use of their images.

\section{Author contribution}

Conceptualization: WL Kuo, SC Chen, JJ Huang. Data curation: WL Kuo, JJ Huang. Formal analysis: CL Su, JR Yang, JJ Huang. Methodology: WL Kuo, SC Chen, DC Cheong, JJ Huang. Project administration: WL Kuo, SC Chen, JJ Huang. Visualization: CL Su, JR Yang, WL Kuo, DC Cheong, JJ Huang. Writing - original draft: CL Su, JR Yang, JJ Huang. Writing - review \& editing: WL Kuo, SC Chen, DC Cheong, JJ Huang.

\section{ORCID}

Chun-Lin Su https://orcid.org/0000-0002-8111-707X Jia-Ruei Yang https://orcid.org/0000-0002-8584-870X Wen-Ling Kuo https://orcid.org/0000-0002-1145-8514 Shin-Cheh Chen https://orcid.org/0000-0002-0177-0045 David Chon-Fok Cheong https://orcid.org/0000-0003-1430-7348

Jung-Ju Huang https://orcid.org/0000-0001-9568-7834

\section{REFERENCES}

1. Sacchini V, Pinotti JA, Barros AC, et al. Nipple-sparing mastectomy for breast cancer and risk reduction: oncologic or technical problem? J Am Coll Surg 2006;203:704-14.

2. Gerber B, Krause A, Dieterich M, et al. The oncological safety of skin sparing mastectomy with conservation of the nipple-areola complex and autologous reconstruction: an extended follow-up study. Ann Surg 2009;249:461-8.

3. Munhoz AM, Aldrighi CM, Montag E, et al. Clinical outcomes following nipple-areola-sparing mastectomy with immediate implant-based breast reconstruction: a 12-year experience with an analysis of patient and breast-related factors for complications. Breast Cancer Res Treat 2013;140: 545-55.

4. Pek WS, Tan BK, Ru Ng YY, et al. Immediate breast reconstruction following nipple-sparing mastectomy in an Asian population: aesthetic outcomes and mitigating nipple-areolar complex necrosis. Arch Plast Surg 2018;45:229-38.
5. Colwell AS, Christensen JM. Nipple-sparing mastectomy and direct-to-implant breast reconstruction. Plast Reconstr Surg 2017;140:44S-50S.

6. de Vita R, Buccheri EM. Nipple sparing mastectomy and direct to implant breast reconstruction, validation of the safe procedure through the use of laser assisted indocyanine green fluorescent angiography. Gland Surg 2018;7:258-66.

7. Boneti C, Yuen J, Santiago C, et al. Oncologic safety of nipple skin-sparing or total skin-sparing mastectomies with immediate reconstruction. J Am Coll Surg 2011;212:686-93.

8. Azouz V, Lopez S, Wagner DS. Surgeon-controlled comparison of direct-to-implant and 2-stage tissue expander-implant immediate breast reconstruction outcomes. Ann Plast Surg 2018;80:212-6.

9. Grover R, Rueda S, Gurunluoglu R. Is single-stage prosthetic reconstruction cost effective? A cost-utility analysis for the use of direct-to-implant breast reconstruction relative to expander-implant reconstruction in postmastectomy patients. Plast Reconstr Surg 2017;139:1204e-1205e.

10. Wang HY, Ali RS, Chen SC, et al. One-stage immediate breast reconstruction with implant following skin-sparing mastectomy in Asian patients. Ann Plast Surg 2008;60:3626.

11. Colwell AS, Damjanovic B, Zahedi B, et al. Retrospective review of 331 consecutive immediate single-stage implant reconstructions with acellular dermal matrix: indications, complications, trends, and costs. Plast Reconstr Surg 2011; 128:1170-8.

12. Vardanian AJ, Clayton JL, Roostaeian J, et al. Comparison of implant-based immediate breast reconstruction with and without acellular dermal matrix. Plast Reconstr Surg 2011; 128:403e-410e.

13. Ho G, Nguyen TJ, Shahabi A, et al. A systematic review and meta-analysis of complications associated with acellular dermal matrix-assisted breast reconstruction. Ann Plast Surg 2012;68:346-56.

14. Gamboa-Bobadilla GM. Implant breast reconstruction using acellular dermal matrix. Ann Plast Surg 2006;56:22-5.

15. Salzberg CA, Ashikari AY, Koch RM, et al. An 8-year experience of direct-to-implant immediate breast reconstruction using human acellular dermal matrix (AlloDerm). Plast Reconstr Surg 2011;127:514-24.

16. Basta MN, Gerety PA, Serletti JM, et al. A systematic review and head-to-head meta-analysis of outcomes following direct-to-implant versus conventional two-stage implant reconstruction. Plast Reconstr Surg 2015;136:1135-44.

17. Lardi AM, Ho-Asjoe M, Mohanna PN, et al. Immediate breast reconstruction with acellular dermal matrix: factors 
affecting outcome. J Plast Reconstr Aesthet Surg 2014;67: 1098-105.

18. Gdalevitch P, Ho A, Genoway K, et al. Direct-to-implant single-stage immediate breast reconstruction with acellular dermal matrix: predictors of failure. Plast Reconstr Surg 2014;133:738e-747e.

19. Roostaeian J, Sanchez I, Vardanian A, et al. Comparison of immediate implant placement versus the staged tissue expander technique in breast reconstruction. Plast Reconstr Surg 2012;129:909e-918e.

20. Susarla SM, Ganske I, Helliwell L, et al. Comparison of clinical outcomes and patient satisfaction in immediate singlestage versus two-stage implant-based breast reconstruction. Plast Reconstr Surg 2015;135:1e-8e.

21. Negenborn VL, Dikmans REG, Bouman MB, et al. Predictors of complications after direct-to-implant breast reconstruction with an acellular dermal matrix from a multicentre randomized clinical trial. Br J Surg 2018;105:1305-12.

22. Hunsicker LM, Ashikari AY, Berry C, et al. Short-term complications associated with acellular dermal matrix-assisted direct-to-implant breast reconstruction. Ann Plast Surg 2017;78:35-40.

23. Petit JY, Veronesi U, Orecchia R, et al. Nipple sparing mastectomy with nipple areola intraoperative radiotherapy: one thousand and one cases of a five years experience at the Eu- ropean institute of oncology of Milan (EIO). Breast Cancer Res Treat 2009; 117:333-8.

24. Algaithy ZK, Petit JY, Lohsiriwat V, et al. Nipple sparing mastectomy: can we predict the factors predisposing to necrosis? Eur J Surg Oncol 2012;38:125-9.

25. Roh TS, Kim JY, Jung BK, et al. Comparison of outcomes between direct-to-implant breast reconstruction following nipple-sparing mastectomy through inframammary fold incision versus noninframammary fold incision. J Breast Cancer 2018;21:213-21.

26. Salzberg CA, Ashikari AY, Berry C, et al. Acellular dermal matrix-assisted direct-to-implant breast reconstruction and capsular contracture: a 13-year experience. Plast Reconstr Surg 2016;138:329-37.

27. Lohmander F, Lagergren J, Roy PG, et al. Implant based breast reconstruction with acellular dermal matrix: safety data from an open-label, multicenter, randomized, controlled trial in the setting of breast cancer treatment. Ann Surg 2019;269:836-41.

28. Colwell AS. Current strategies with 1-stage prosthetic breast reconstruction. Gland Surg 2015;4:111-5.

29. Spear SL, Sher SR, Al-Attar A. Focus on technique: supporting the soft-tissue envelope in breast reconstruction. Plast Reconstr Surg 2012;130(5 Suppl 2):89S-94S. 